\title{
Development In Vivo and In Vitro to Blastocysts of Porcine Oocytes Matured and Fertilized In Vitro
}

\author{
Naomi KASHIWAZAKI ${ }^{1)}$, Kazuhiro KIKUCHI'), Kaoru SUZUKI ${ }^{1,2}$, \\ Junko NOGUCHI ${ }^{2)}$, Takashi NAGAI'), Hiroyuki KANEKO ${ }^{2)}$ and Masao SHINO') \\ 1) Laboratory of Animal Reproduction, School of Veterinary Medicine, Azabu University, \\ Sagamihara, Kanagawa 229-8501, Japan, 2) National Institute of Agrobiological Sciences, \\ Kannondai 2-1-2, Tsukuba, Ibaraki 305-8602, Japan
}

\begin{abstract}
The objective of the present study was to evaluate the development to the blastocyst stage in vivo and in vitro of porcine oocytes matured and fertilized in vitro (IVM/IVF). When IVM/IVF oocytes had been transferred to oviducts of synchronized recipients just after IVF, more than $70 \%$ of blastocysts recovered after 6 days of transfer had developed to the hatched blastocyst stage with a mean cell number of 181.5 per blastocyst. In contrast, when IVM/IVF oocytes were cultured in vitro for 2 days and then transferred to the oviducts of recipients, or cultured in vitro for 6 days without transfer to oviducts, most recovered blastocysts were still enclosed by zona pellucidae. Mean cell numbers of blastocysts obtained from IVM/IVF oocytes cultured for 2 days in vitro plus 4 days in the oviducts and for 6 days in vitro decreased significantly $(\mathrm{P}<0.01)$ to 58.2 and 38.4, respectively, compared with that of blastocysts obtained from IVM/IVF oocytes cultured in oviducts for 6 days. These results suggest that porcine IVM/IVF oocytes have a high potential for developing to the blastocyst stage equal to that of in vivo matured oocytes and that the in vitro culture conditions used for IVM/IVF oocytes in the present study were suboptimal.

Key words: Porcine, Oocyte, IVM/IVF, Embryo transfer, Blastocyst
\end{abstract}

(J. Reprod. Dev. 47: 303-310, 2001)

$\mathbf{T}$ ransgenic technologies including gene manipulations and nuclear transfer in pigs will greatly improve pork production and allow a number of new biomedical applications such as xenotransplantation in humans and bioreactors for industry in the near future $[1,2]$. When porcine oocytes/embryos are manipulated to generate transgenic pigs, most frequently oocytes/embryos are surgically collected from donor females, but this is a very costly and laborious technique [3]. If we could establish a reliable in vitro production (IVP) system for porcine embryos, it would be more effective, less costly and time-consuming. But IVP systems for pigs suffer from a low rate of

Accepted for publication: August 22, 2001

Correspondence: N. Kashiwazaki development of IVM/IVF oocytes to the blastocyst stage [4]. In general, the competence of porcine oocytes/embryos derived in vitro is lower than those derived in vivo [3]. Efforts to produce porcine embryos in vitro have focused on the establishment of a system for efficient IVM and IVF of oocytes [5]. Recently, a successful porcine cloning IVM system was reported [6]. In that system, IVM oocytes were used as recipient oocytes resulting in live offspring. However, the developmental potential of IVM oocytes was not discussed.

The objective of the present study was to evaluate the developmental ability to the blastocyst stage, in vivo and in vitro of porcine IVM/IVF oocytes. We also compared the quality of the IVM/ IVF-derived blastocysts, which were developed in 
vivo and in vitro.

\section{Materials and Methods}

\section{Oocyte collection and IVM}

Porcine ovaries were obtained from prepubertal cross-bred (Landrace, Large White and Duroc breeds) gilts at a local slaughterhouse and were transported to the laboratory at $35 \mathrm{C}$. Cumulusoocyte complexes (COCs) were collected from follicles of 3-5 mm in diameter in Medium 199 (with Hanks' salts, Gibco, Life Technologies Inc., Grand Island, NY, USA) supplemented with $10 \%$ (v/v) fetal bovine serum (Gibco), 20 mM HEPES (Dojindo Laboratories, Kumamoto, Japan), 100 unit/ml penicillin $\mathrm{G}$ potassium (Sigma Chemical Co., St. Louis, MO, USA) and $0.1 \mathrm{mg} / \mathrm{ml}$ streptomycin sulfate (Sigma) [7]. IVM of oocytes was carried out as reported previously [8]. In brief, collected COCs were cultured in modified NCSU37 solution [9] containing $10 \%(\mathrm{v} / \mathrm{v})$ porcine follicular fluid, $0.6 \mathrm{mM}$ cysteine, $1 \mathrm{mM}$ dibutytyl cyclic AMP (dbcAMP, Sigma), 10 IU/ml eCG (PMS 1,000 IU, Nihon Zenyaku Kogyo, Koriyama, Japan), $10 \mathrm{IU} / \mathrm{ml}$ hCG (Puberogen 500 unit, Sankyo, Tokyo, Japan), and the antibiotics mentioned above for 20-22 h; followed by culture in modified NCSU37 solution without dbcAMP and hormones for 24 h. The maturation culture was performed at $39 \mathrm{C}$ under $5 \% \mathrm{CO}_{2}$ in humidified air.

\section{IVF of IVM oocytes}

Boar epididymides from the Meishan breed were collected. Epididymal spermatozoa were collected and frozen as described previously [10]. IVF was carried out as described previously [8]. Briefly, spermatozoa were thawed and preincubated for $1 \mathrm{~h}$ at $37 \mathrm{C}$ in Medium 199 adjusted to $\mathrm{pH} 7.8$ [11]. A portion $(10 \mu \mathrm{l})$ of preincubated spermatozoa was introduced into the fertilization medium, $90 \mu \mathrm{l}$ Bracket and Oliphant solution [12] supplemented with $10 \mathrm{mg} / \mathrm{ml}$ bovine serum albumin (BSA, Fraction V, Sigma) containing 10 IVM oocytes surrounded by expanded cumulus cells. The final sperm concentration was $1 \times 10^{6} / \mathrm{ml}$. Insemination was carried out for $5 \mathrm{~h}$. To examine the IVF results, some inseminated oocytes were transferred to the in vitro culture (IVC) medium, NCSU-37 solution containing $4 \mathrm{mg} / \mathrm{ml}$ BSA and $50 \mu \mathrm{M} \beta$ mercaptoethanol, and subsequently cultured at
$38.5 \mathrm{C}$ under conditions of $\mathrm{O}_{2}, \mathrm{CO}_{2}$ and $\mathrm{N}_{2}$ adjusted to $5 \%, 5 \%$ and $90 \%$, respectively, for $5 \mathrm{~h}$ [13]. They were then fixed with acetic alcohol (1:3), stained with $1 \%$ aceto-orcein and examined under a phase contrast microscope.

\section{In vivo and in vitro culture of IVM/IVF oocytes}

Early pregnant crossbred gilts were used as embryo recipients. Estrus synchronization was carried out by an intramuscular injection of $0.18 \mathrm{mg}$ prostaglandin $\mathrm{F}_{2 \alpha}$ analogue (Cloprostenol, Planate, Sumitomo Seiyaku, Osaka, Japan) on the 16th to 45 th day of gestation, followed by a second injection of $0.09 \mathrm{mg}$ Cloprostenol $24 \mathrm{~h}$ later $[8,14]$. One thousand IU of eCG (PMS 1,000 Tani NZ, Nihon Zenyaku, Kohriyama, Japan) was administered intramuscularly at the same time as the second Cloprostenol injection. Ovulation was induced by intramuscular injection of 500 IU hCG (Puberogen, Sankyo, Tokyo, Japan) $72 \mathrm{~h}$ after the eCG injection. IVM/IVF oocytes were transferred to NCSU-37 supplemented with $4 \mathrm{mg} / \mathrm{ml} \mathrm{BSA}$ and 25 mM HEPES, the osmolarity of which had been adjusted to $280 \mathrm{mOsm}$. After confirmation of immobility of spermatozoa, 200 IVM/IVF oocytes per recipient without selection were surgically introduced into the oviducts of recipients $43-45 \mathrm{~h}$ after the hCG injection (Day 0) and, on the 6th day (Day 6) after IVF, the uterine horns of the recipients receiving IVM/IVF oocytes were surgically flushed with $50 \mathrm{ml}$ Dulbecco's phosphate buffered saline containing $1 \%(\mathrm{v} / \mathrm{v})$ fetal bovine serum (in vivo group). Additionally, IVM/IVF oocytes were subsequently cultured in vitro for 2 days in the IVC medium at $38.5 \mathrm{C}$ under conditions of $\mathrm{O}_{2}, \mathrm{CO}_{2}$ and $\mathrm{N}_{2}$ adjusted to $5 \%, 5 \%$ and $90 \%$, respectively. Then, the cleaved embryos obtained from the culture of 200 IVM/IVF oocytes per recipient were surgically transferred into the oviducts of recipients at 93-95 $\mathrm{h}$ after the hCG injection (Day 2). The uterine horns were flushed on Day 6 (IVC plus in vivo group). IVM/IVF oocytes were also cultured in vitro in the IVC medium for 6 days (IVC group).

\section{Evaluation of embryonic development}

Recovered embryos and oocytes were evaluated for developmental stage and morphology under an inverted microscope. An embryo having a clear blastocoel was defined as a blastocyst. And the blastocysts were classified into 3 stages: 1) blastocyst stage including expanded blastocyst 
stage, 2) hatching blastocyst stage, and 3) hatched blastocyst stage. The blastocysts were then fixed and stained. For the IVC group, blastocysts were fixed and stained in the same manner. The developmental potential of IVM/IVF oocytes and the efficiency of in vivo culture systems were evaluated by the blastocyst formation rates and by the numbers of cells in a resulting blastocyst which was regarded as embryo quality.

\section{Statistical analysis}

Data after transformation using arcsine of percentage of blastocyst formation [15] and data for mean cell numbers per blastocyst were subjected to analysis of variance using GLM procedures of Statistical Analysis System (SAS), and were analyzed by the Duncan's multiple range test.

\section{Results}

\section{IVM and IVF}

In the present study, about $75 \%$ of examined oocytes were identified as matured to the second metaphase. As shown in Table 1, the percentage of sperm penetrating to the examined oocytes was about $40 \%$, and almost all of the penetrated oocytes were activated. The percentage of monospermy was $15.4 \%$ and all of the monospermic oocytes formed a well-developed male pronucleus. These monospermic oocytes were judged as normally fertilized because they had single male and female pronuclei with 2 polar bodies.

\section{Blastocyst development in vivo and in vitro}

When a total of 1,400 IVM/IVF oocytes were transferred just after IVF into oviducts of 7 recipient gilts (200 per recipient, in vivo group), a total of 606 oocytes/embryos were recovered from them on Day 6. Blastocysts (total 156) were recovered from 4 recipients that received IVM/IVF oocytes, however only degenerated or fragmented oocytes were obtained from the other 3 recipients. The recovery rate in the 7 recipients that received IVM/ IVF oocytes on Day 0 to the total number of the transferred oocytes was $43.3 \%(606 / 1,400)$. In contrast, when all of the cleaved embryos produced from 2 days in vitro culture of 1,000 IVM/IVF oocytes (572) were transferred to 5 recipients (IVC plus in vivo group), the recovery rate from the 5 recipients of the cleaved embryos was 61.9\% (354/ 572). Blastocysts (total 15) were recovered from 3 recipients. As shown in Table 2, when blastocysts collected from the in vivo group (total 156) were

Table 1. In vitro fertilization (IVF) of porcine oocytes matured in vitro

\begin{tabular}{|c|c|c|c|}
\hline \multirow{2}{*}{$\begin{array}{l}\text { Total number } \\
\text { of oocytes } \\
\text { examined }\end{array}$} & \multicolumn{3}{|c|}{ No. (\%) of normal fertilization ${ }^{a}$} \\
\hline & Penetration & $\mathrm{MPN}^{\mathrm{b}}$ formation & Monospermy ${ }^{\mathrm{c}}$ \\
\hline 78 & $32(41.0)$ & $31(39.7)$ & $12(15.4)$ \\
\hline \multicolumn{4}{|c|}{$\begin{array}{l}\text { a Penetrated oocytes with one first and one second polar body } \\
{ }^{\text {b }} \text { MPN; male pronucleus. } \\
\text { c With both single MPN and female pronucleus. } \\
\text { Three replicated trials were carried out. }\end{array}$} \\
\hline
\end{tabular}

Table 2. Development of porcine IVM/IVF oocytes to blastocysts after transfer to recipients on Day 0 (IVF) and Day 2 of IVF or culture in vitro for 6 days

\begin{tabular}{lcccccc}
\hline \multirow{2}{*}{$\begin{array}{c}\text { Developmental } \\
\text { environment }\end{array}$} & $\begin{array}{c}\text { Total } \\
\text { No. } \\
\text { collected }\end{array}$ & $\begin{array}{c}\text { Blastocysts } \\
\text { (Total) }\end{array}$ & $\begin{array}{c}\text { Blastocyst } \\
\text { stage }\end{array}$ & $\begin{array}{c}\text { Hatching } \\
\text { blastocyst stage }\end{array}$ & $\begin{array}{c}\text { Hatched } \\
\text { blastocyst stage }\end{array}$ & $\begin{array}{c}\text { Degenerated } \\
\text { and fragmented } \\
\text { embryos }\end{array}$ \\
\hline In vivo & 421 & $156(37.1)^{\mathrm{e}}$ & $32(7.6)$ & $9(2.1)$ & $115(27.3)^{\mathrm{e}}$ & $265(62.9)^{\mathrm{e}}$ \\
${\text { IVC plus } i n v i v o^{\mathrm{c}}}^{\mathrm{e}}$ & 318 & $15(4.7)^{\mathrm{f}}$ & $13(4.1)$ & - & $2(0.6)^{\mathrm{f}}$ & $303(95.3)^{\mathrm{f}}$ \\
IVC $^{\mathrm{d}}$ & 254 & $51(20.1)$ & $51(20.1)$ & - & - & $203(79.9)$ \\
\hline
\end{tabular}

a The blastocyst stage includes expanded blastocysts with intact zona pellucidae.

b Total 1,400 IVM/IVF oocytes were transferred to 7 recipients on Day 0 (in vivo) and blastocysts were recovered from 4 recipients on Day 6.

${ }^{\mathrm{c}}$ Total 572 embryos (cleaved embryos) produced in vitro were transferred to 5 recipients on Day 2 (IVC plus in vivo) and blastocysts were recovered from 3 recipients on Day 6.

${ }^{\mathrm{d}}$ IVM/IVF oocytes were subsequently cultured in vitro without transfer until Day 6 of IVF. Four replicated trials were carried out.

e,f Different superscripts show significant difference between in vivo and IVC plus in vivo groups in the same column $(\mathrm{P}<0.05)$. 
a

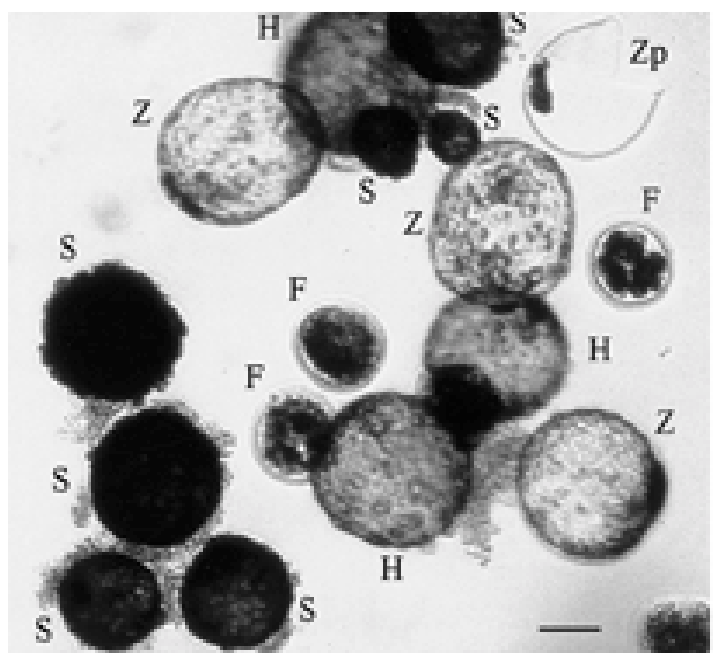

b

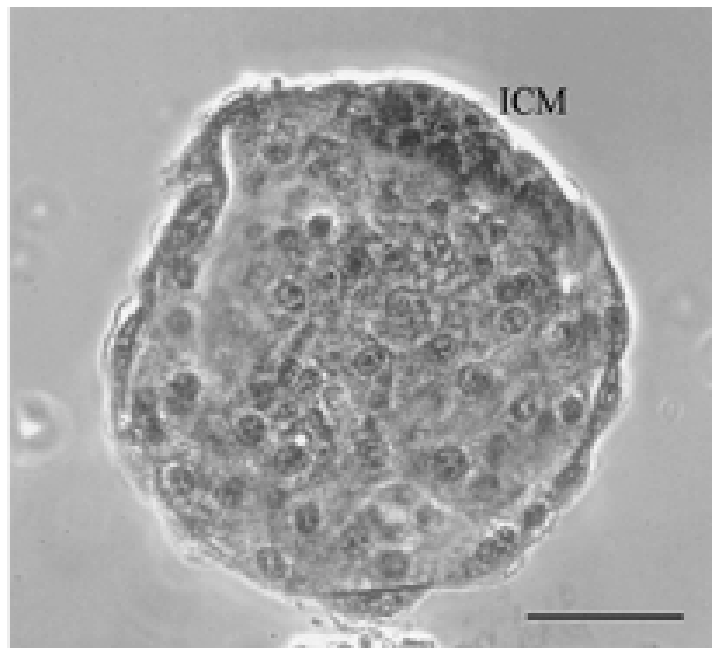

Fig. 1. Porcine embryos derived from IVM/IVF oocytes and developed in vivo after IVF. Porcine oocytes were matured and fertilized in vitro, then transferred into oviducts of synchronized recipients without culture and recovered from uterine horns of the recipients on Day 6. a) Embryos at various stages were recovered. $\mathrm{Z}$; expanded blastocysts with intact zona pellucidae. H and S; hatched blastocysts, the former is shown just after hatching and the latter is shown after shrinkage. F; fragmented oocyte or embryo. Zp; zona pellucida after hatching. Note; hatching blastocyst is out of focal area. b) One of the hatched blastocysts was fixed, stained and counted for the cell number. The cell number of the embryo was 198. Inner cell mass (ICM) was seen clearly. Bar $=100 \mu \mathrm{m}$.

classified under the microscope for their developmental stage, $73.7 \%(115 / 156)$ of them had already hatched. Some of the hatched blastocysts were shrunken at the time of embryo collection (Fig. 1). When blastocysts from the IVC plus in vivo group (total 15) were classified, most $(86.7 \%, 13 / 15)$ of the recovered blastocysts were enclosed with zona pellucidae. When IVM/IVF oocytes were cultured in vitro for 6 days (IVC group), 20.1\% (51/ 254) of them developed to the blastocyst stage with an evident blastocoel, but all of them were just expanded, and none proceeded to hatch (Fig. 2). The hatching rate of the in vivo group was significantly $(\mathrm{P}<0.05)$ higher than that of the IVC plus in vivo group.

Blastocysts developed in vivo and in vitro were immediately fixed, stained and evaluated for embryo quality by counting cell numbers. As shown in Table 3, the mean cell number calculated from all blastocysts derived from the in vivo group was significantly higher $(\mathrm{P}<0.01)$ than those of blastocysts obtained from the IVC pulse in vivo and IVC groups.

\section{Discussion}

Our previous study [8] suggested that the viability of in vitro produced porcine embryos is decreased by IVC after IVF, because the developmental rates to fetuses on day-29 of gestation or live new born piglets decreased with increasing length of IVC period. However, it was not clear at which developmental stage in vitro produced embryos were affected by IVC. When IVM/IVF oocytes were transferred on Day 0 without IVC and subjected to in vivo conditions until Day 6 in the present study (in vivo group), advanced incidences of embryonic development to the blastocyst stages, blastocyst, hatching blastocyst or hatched blastocyst, were observed. Especially, the developmental rate to the hatched blastocyst stage in the in vivo group was significantly higher, and the mean cell number of the hatched blastocysts was 215.0. The mean cell number of the blastocysts in the in vivo group was 181.5 , nearly equal to that of blastocysts derived in vivo at the same gestation period in the pig, which was reported to be hatching to hatched blastocysts containing 120 to 480 cells $[16,17]$. In contrast, poor 
a

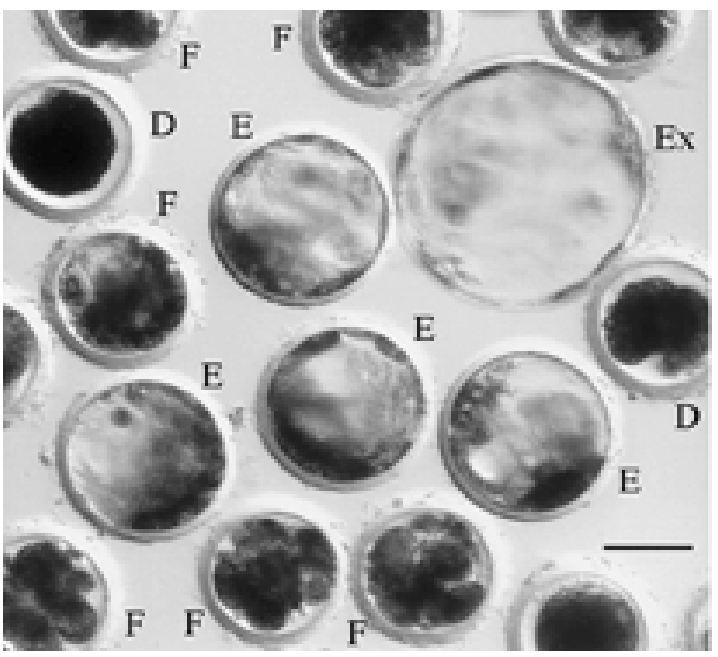

b

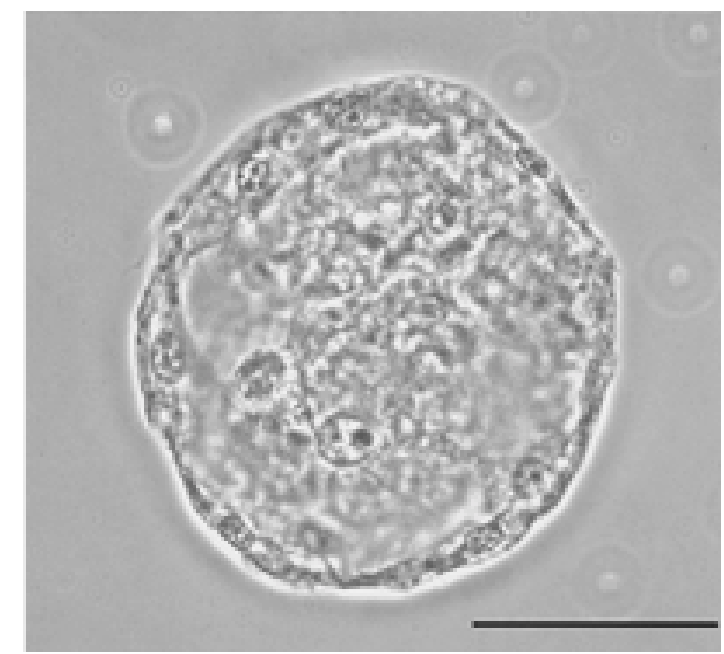

Fig. 2. Porcine embryos derived from IVM/IVF oocytes and developed in vitro after IVF. Porcine oocytes were matured, fertilized and then cultured in vitro for 6 days. a) Blastocysts with intact zona pellucidae were obtained. E; expanded blastocyst. Ex; large expanded blastocyst. Hatching or hatched blastocysts were not observed. D; degenerated oocyte or embryo. F; fragmented oocyte or embryo. b) One of the expanded blastocysts was fixed, stained and counted for the cell number. The cell number of the embryo was 45 . Inner cell mass was not identified in the expanded blastocyst developed in vitro. Bar $=100 \mu \mathrm{m}$.

Table 3. Numbers of cells of porcine blastocysts developed in vivo, in vitro plus in vivo, or in vitro after IVM/IVF

\begin{tabular}{llccc}
\hline & \multicolumn{4}{c}{ Mean cell numbers per blastocyst ${ }^{\mathrm{a}}$} \\
\cline { 2 - 4 } $\begin{array}{c}\text { Developmental } \\
\text { environment }\end{array}$ & Blastocyst $^{\mathrm{a}}$ & $\begin{array}{c}\text { Hatching } \\
\text { blastocyst }\end{array}$ & $\begin{array}{c}\text { Hatched } \\
\text { blastocyst }\end{array}$ & All blastocysts \\
\cline { 2 - 5 } $\begin{array}{l}\text { In vivo } \\
\text { IVC plus in vivo }\end{array}$ & $58.4 \pm 9.3^{\mathrm{b}}$ & $134.0 \pm 21.0$ & $215.0 \pm 9.0^{\mathrm{f}}$ & $181.5 \pm 8.4^{\mathrm{d}}$ \\
IVC $^{\mathrm{c}}$ & $38.4 \pm 3.2^{\mathrm{e}}$ & - & $58.8 \pm 18.5^{\mathrm{g}}$ & $58.2 \pm 9.1^{\mathrm{e}}$ \\
\hline
\end{tabular}

Mean \pm SEM.

a Blastocysts shown in Table 2 were fixed, stained and counted for the cell numbers.

${ }^{\mathrm{b}}$ Embryos produced in vitro were transferred to recipients at Day 0 (in vivo) or Day 2 (IVC plus in vivo) after IVF, and were recovered on Day 6. IVF was carried out on Day 0.

${ }^{c}$ IVM/IVF oocytes were cultured in vitro until Day 6.

$\mathrm{d}, \mathrm{e}$ Values in the same column with different superscripts differ significantly $(\mathrm{P}<0.01)$.

$\mathrm{f}, \mathrm{g}$ Values in the same column with different superscripts differ significantly $(\mathrm{P}<0.05)$.

developmental ability and low cell number (58.2) of the blastocysts, which were cultured for 2 days and transferred (IVC plus in vivo group), were observed. This is consistent with the report of Yoshida et al. [18] which showed that the cell number of porcine in vitro produced embryos transferred to recipient females at $33 \mathrm{~h}$ after IVF $(2$ cell-stage embryos) and recovered from them on
Day 6 was 65.8. The detrimental effect of IVC was evident, when IVP embryos were cultured for 6 days without transfer to recipients. The blastocysts showed a smaller cell number (38.4), and this cell number is almost equal to that of our previous study $(39,[8])$ or those reported by others (30 [19] and $37[20])$.

It was also reported [21] that, when porcine 4-cell 
stage embryos obtained in vivo were cultured in vitro and developed to the blastocyst stage, their mean cell number was 34 , however, when early blastocysts derived in vivo were cultured in vitro and developed to the hatching blastocyst stage, the cell number increased to 151 . This fact emphasizes the incompetence of the culture system even when in vivo produced embryos are used. Although the culture systems used in each study were not the same, the results generally suggest that the culture systems for porcine zygotes/embryos are not optimal, but that IVM/IVF oocytes can develop to the blastocyst stage when they are placed in optimal circumstances such as in suitable female reproductive tracts. In the present study, $37.1 \%$ of the IVM/IVF oocytes developed in vivo to the blastocyst stage. The proportion was higher than that of the IVC group, and seems to be higher than the monospermic fertilization rate $(15.3 \%)$. Although polyspermy of the egg cytoplasm frequently occurs, polyspermic oocytes can develop to the blastocyst stage in the pig $[22,23]$. Thus, some of the blastocysts in the in vivo group might be derived from polyspermic oocytes.

On the other hand, considering the fact that the recovery rate for the IVC plus in vivo group (61.9\%) was higher than that for the in vivo group (43.3\%), in the present study, it seems that more degenerated or fragmented embryos might be eliminated from the uteri of recipients with increasing length of time after transfer of IVM/IVF oocytes. No blastocyst was obtained from 5 of 12 recipients in the present study. Ovulation and formation of corpus luteum were confirmed in all the recipients at the time of embryo transfer and recovery, respectively. This means that synchronization of estrus with hormone administration seemed to have occurred. However, the circumstances around the transferred embryos (oviductal circumstances) might be insufficient for supporting embryonic development in vivo. Oviductal epithelium itself or its secretary product(s) enhances in vitro embryonic development in pigs $[24,25]$. Synchronization of oviductal conditions may not occur in recipients resulting in a failure of embryonic development. In addition, the embryos cultured in vitro for the first 2 days after IVF had a decreased developmental ability to the blastocyst stage. Our previous data [8] also showed that the developmental ability of IVM/IVF oocytes to fetuses or piglets decreased once they had been cultured in vitro for 2 days before transfer to the oviducts. These results suggest that the oviductal circumstances are important for early cleavage embryos to complete their embryonic development. The first 2 days after IVF seem to be the prerequisite stage for embryonic genome activation [26]. Thus, we suggest that oviductal circumstances might be related to embryonic potential after genome activation.

In vivo matured oocytes are widely used for biotechnologies including cloning by nuclear transfer [27, 28], because porcine in vivo matured oocytes seem to have excellent developmental ability when compared to IVM oocytes [29]. Recently, the successful porcine cloning by somatic cell nuclear transfer using enucleated IVM oocytes as recipients for the donor nuclei was reported [6]. The results of the present study indicate that the developmental potential of porcine IVM oocytes seems to be almost the same as that of in vivo matured oocytes. IVM oocytes produced in the present study could be used as recipient oocytes for cloning. However, the population of competent oocytes after IVM should be investigated, since all of the IVM oocytes might not have the ability to develop completely as reported previously [29]. It is necessary to establish an efficient method to select oocytes with an excellent ability for development, in other words, a method which is able to distinguish oocytes with complete cytoplasmic maturation as well as nuclear maturation from those matured incompletely. This method will be essential for the successful generation of cloned pigs using in vitro materials.

In conclusion, porcine IVM oocytes have the same developmental potential as those of in vivo matured oocytes suggesting financial advantages for using IVM oocytes as recipient oocytes in cloning. An improved IVC system for IVM/IVF oocytes in pigs may generate advances in this field.

\section{Acknowledgments}

The authors would like to thank Dr. A. Onishi for providing us with Meishan epididymides, Dr. $\mathrm{H}$. Takeda for statistical analysis, Ms. T. Aoki and Ms. E. Yamauchi for technical assistance, and Mr. K. Takagi of Sunrise Farm for preparing recipient gilts. This research was supported in part by Grants-in-Aid Scientific Research (No. 11356008 
and No. 10556059) from the Ministry of Education, Science, Sports and Culture of Japan, and a grant from the Science Research Promotion Fund of the
Promotion and Mutual Aid Corporation for Private Schools of Japan.

\section{References}

1. Wall RJ. Transgenic livestock; Progress and prospects for the futture. Theriogenology 1996; 45: 5768.

2. Prather RS. Pigs is pigs. Science 2000; 289: 18861887.

3. Kashiwazaki N, Shino M. Ability of in vitro manipulated porcine embryos to develop to piglets. J Reprod Dev 2001; 47 (suppl 1): S35-S39.

4. Nagai T, Yamauchi N, Kikuchi K. Nuclear and cytoplasmic maturation in vitro of porcine oocytes. J Reprod Dev 2001; 47 (suppl 1): S55-S62.

5. Funahashi H, Day BN. Advances in in vitro production of pig embryos. J Reprod Fertil 1997; 52 (suppl): 271-283.

6. Betthauser J, Forsberg E, Augenstein M, Childs L, Eilertsen K, Enos J, Forsythe T, Golueke P, Jurgella G, Koppang R, Lesmeister T, Mallon K, Mell G, Misica P, Pace M, Pfister-Genskow M, Strelchenko N, Voelker G, Watt S, Thompson S, Bishop M. Production of cloned pigs from in vitro systems. Nature Biotech 2000; 18: 1055-1059.

7. Kikuchi K, Nagai T, Motlik J, Shioya Y, Izaike Y. Effect of follicle cells on in vitro fertilization of pig follicular oocytes. Theriogenology 1993; 39: 593-599.

8. Kikuchi K, Kashiwazaki N, Noguchi J, Shimada A, Takahashi R, Hirabayashi M, Shino M, Ueda M, Kaneko H. Developmental competence, after transfer to recipients, of porcine matured, fertilized, and cultured in vitro. Biol Reprod 1999; 60: 336-340.

9. Petters RM, Wells KD. Culture of pig embryos. J Reprod Fertil 1993; 48 (suppl): 61-73.

10. Kikuchi K, Nagai T, Kashiwazaki N, Ikeda H, Noguchi J, Shimada A, Soloy E, Kaneko H. Cryopreservation and ensuing in vitro fertilization ability of boar spermatozoa from epididymides stored at $4^{\circ} \mathrm{C}$. Theriogenology 1998; 50: 615-623.

11. Nagai $T$, Takahashi $T$, Masuda $H$, Shioya $Y$, Kuwayama M, Fukushima M, Iwasaki S, Hanada A. In-vitro fertilization of pig oocytes by frozen boar spermatozoa. J Reprod Fertil 1988; 84: 585-591.

12. Bracket BG, Oliphant G. Capacitation of rabbit spermatozoa in vitro. Biol Reprod 1975; 12: 260-274.

13. Kikuchi K, Kashiwazaki N, Noguchi J, Shimada A, Kaneko H. Culture conditions under Low oxygen or with $\beta$-mercaptoethanol enhance embryonic development of porcine zygotes produced in vitro. In: Program of the Fifth International Conference of Pig Reproduction; 1997; Karekulad, the Netherlands (abstract 229).
14. Kashiwazaki N, Nagashima H, Ashman RJ, Christopher GG, Nottle MB. Freezing of in vivo derived and in vitro pre-cultured porcine blastocyst: Differences of the cryoprotective effect between glycerol and ethylene glycol. J Reprod Dev 1996; 42: 193-197.

15. Snedecor GW, Cochran WG. Failures in the assumptions. In: Snedecor GW and Cochran WG (eds.), Statistical Methods, 8th ed. Iowa: The Iowa State University Press; 1989: 273-296.

16. Davis DL. Culture and storage of pig embryos. J Reprod Fertil 1985; 33 (suppl): 115-124.

17. Papaioannou VE, Ebert KM. The preimplantation pig embryo: cell number and allocation to trophectoderm and inner cell mass of the blastocyst in vivo and in vitro. Development 1988; 102: 793-803.

18. Yoshida M, Ishizaki Y, Kawagishi H. Blastocyst formation by pig embryos resulting from in-vitro fertilization of oocytes matured in vitro. J Reprod Fertil 1990; 88: 1-8.

19. Abeydeera LR, Day BN. Fertilization and subsequent development in vitro of pig oocytes inseminated in a modified Tris-buffered medium with frozen-thawed ejaculated spermatozoa. Biol Reprod 1997; 57: 729-734.

20. Wang WH, Abeydeera LR, Cantley TC, Day BN. Effect of oocyte maturation media on development of pig embryos produced by in vitro fertilization. $J$ Reprod Fertil 1997; 111: 101-108.

21. Niemann H, Illera MJ, Dziuk PJ. Developmental capacity, size and number of nuclei in pig embryos cultured in vitro. Anim Reprod Sci 1983; 5: 311-321.

22. Han YM, Abeydeera LR, Kim JH, Moon HB, Cabot RA, Day BN, Prather RS. Growth retardation of inner cell mass cells in polyspermic porcine embryos produced in vitro. Biol Reprod 1999; 60: 1110-1113.

23. Han $\mathbf{Y M}$, Wang $\mathbf{W H}$, Abeydeera LR, Petersen AL, Kim JH, Murphy C, Day BN, Prather RS. Pronuclear location before the first cell division determines ploidy of polyspermic pig embryos. Biol Reprod 1999; 61: 1340-1346.

24. Bureau M, Bailey JL, Sirard MA. Influence of oviductal cells and conditioned medium on porcine gametes. Zygote 2000; 8: 139-144.

25. Kouba A, Abeydeera LR, Alvarez IM, Day BN, Buhi WC. Effects of the porcine oviduct-specific glycoprotein in fertilization, polyspermy, and embryonic development in vitro. Biol Reprod 2000; 
63: $242-250$.

26. Kopecny V. High-resolution autoradiographic studies of comparative nucleologenesis and genome reactivation during early embryogenesis in pig, man and cattle. Reprod Nutr Dev 1989; 29: 589-600.

27. Onishi A, Iwamoto M, Akita T, Mikawa S, Takeda K, Awata T, Hanada H, Perry ACF. Pig cloning by microinjection of fetal fibroblast nuclei. Science 2000; 289: 1188-1190.
28. Polejaeva IA, Chen SH, Vaught TD, Page RL, Mullins J, Ball S, Dai Y, Boone J, Walker S, Ayares DL, Colman A, Campbell KHS. Cloned pigs produced by nuclear transfer from adult somatic cells. Nature 2000; 407: 86-90.

29. Nagashima H, Grupen CG, Ashman RJ, Nottle MB. Developmental competence of in vivo and in vitro matured porcine oocytes after subzonal sperm injection. Mol Reprod Dev 1996; 45: 359-63. 